our investigation, surface ECG showing a slow atrioventricular junctional rhythm in the absence of $\mathrm{P}$ waves and the atria could not be stimulated on invasive EPS in four of the affected patients. Other two younger patients had a lower $\mathrm{P}$ wave without atrial mechanical activity. The proband as well as her two nephews had ventricular pacemaker implantation. ECGs were also recorded in a further 38 members of this family, but all other relatives appeared unaffected. The genetic basis for familial AS is unknown. Candidate gene were analysed by DNA sequencing, but no missense mutation could be found. This suggests that polymorphic genetic inheritance may exist in familial AS.

\title{
Other
}

\section{[gw22-e0425] GENETIC STUDY OF FAMILIAL ATRIAL} STANDSTILL

Li Hailing, Peng Wenhui, Tang Kai, Zhao Dongdong, Weiyi Dong, Li Weiming, Xu Yawei The No.10 People's Hospital of Shanghai

10.1136/heartjnl-2011-300867.726

Atrial standstill (AS) is an extremely rare arrhythmia, characterised by the absence of electrical and mechanical activity in the atria. The authors hereby present a large family with clustering of AS. Forty-four family members were clinically evaluated. Six patients were unambiguously affected by AS. They are the proband, her daughter, two of her nephews, one of her niece and her niece's son. Based on the pedigree, it maybe conforms to autosomal dominant inheritance. Their clinical histories were very similar, first presented with symptoms of syncope in their early twenties and symptoms worsened with age. Before total AS, atrial bradycardia, atrial fibrillation, atrioventricular block could be found in surface ECG. In 